\title{
Design of Intelligent Home System Based on Single Chip Microcomputer Zijian Zhang ${ }^{\mathrm{a}}$, Hongtao $\mathrm{Yu}^{\mathrm{b}^{*}}$, Yanhua Lei ${ }^{\mathrm{c}}$ and Datong Cui ${ }^{\mathrm{d}}$ College of Automation, Shenyang Institute of Engineering, Shenyang Liaoning110136, China \\ a384495501@qq.com, bneu970773@sohu.com, 'yhw127@163.com, dcuidatong9558@sohu.com
}

Keywords: Intelligent home system; Single chip microcomputer; Arduino; Multi-parameters; Monitoring

\begin{abstract}
Aiming at connecting home appliances, monitoring residential environment and achieving portability, a smart home environmental monitoring platform was designed based on Arduino MCU to monitor residential temperature, humidity and illuminance, by selecting environmental parameter sensors, designing and implementing of monitoring hardware and software. After the experiment analysis and debugging verification, the monitor designed in this paper can run normally, accurately and quickly, collecting various environmental information and giving feedback, overcoming the various drawbacks of the traditional monitoring system.
\end{abstract}

\section{Introduction}

As the idea of information technology popularized, people now hope to integrate various smart devices into their home environment, which can connect both their home appliances and transfer residential environmental data through ubiquitous networks. With the combination of appropriate hardware and software, the smart devices in home environment[1-3] can achieve the function of connecting objects and home appliances through network. In the meantime, various environmental factors in the home environment are also collected and transfer through the network, satisfying people's requirements for controlling home appliances through the network and monitoring their home environment, which are traditionally hard to achieve. The concept of smart home combines the traditional home environment concept with modern electronic information technologies such as wireless communication technology, intelligent control technology, and the internet of things to achieve intelligent home, improving the safety, efficiency, comfort, and convenience of the traditional residential environment. Currently, the majority of smart home systems lacks in the capability of monitoring multiple parameters simultaneously and portability. This article introduces a new environmental monitoring system with the capability of monitoring environmental factors of multi-parameters, such as wind speed, humidity, and temperature, in a synchronized fashion based on the Arduino MCU and I/O extension modules that are compatible with the platform, by coding and the circuit board designing. The platform is also capable of uploading gathered data to an Android platform acting as a host machine.

\section{The Design Idea}

This article designs a portable multi-parameter environmental monitoring device with a MCU as its core, by selecting the appropriate sensors to collect and sample the target parameters. The output is then transformed into the appropriate 16-bit dataset and fed into the MCU. After the data is processed, it is transferred to the host machine, where it is displayed in an appropriate manner. In the meantime, the process of software and hardware design and debugging should also be conducted. Program design process is shown in Fig. 1.

Multi-Parameters Monitoring. The user set the number of monitoring groups to be collected and the time interval between each of the collection during the initialization phase of the system. After the initialization, the system begins collecting the various data and transfer the collected data set to host machine via Bluetooth communication, for it to process and record the data. In addition, the data that the parameter monitoring sensor transmits to the Arduino MCU is not in the desired format. For 
instance, the data returned by the temperature sensor appears in a value between 0 and 1024 . These values would be processed based on mathematical formulas and models to scientific unit. The data is then fed back to the computer via serial communication. Specific monitoring parameters[4,5] are as follows:

(1) Temperature

(2) Humidity

(3) Wind speed

Serial Port Feedback. In the process of coding, it is necessary to ensure that the host machine can receive feedback from Arduino and display it on the serial port monitor. Since the Arduino platform is based on the $\mathrm{C}$ language, the grammar itself includes serial communication, hence $9600 \mathrm{kbp}$ serial communication can be achieved directly through programming.

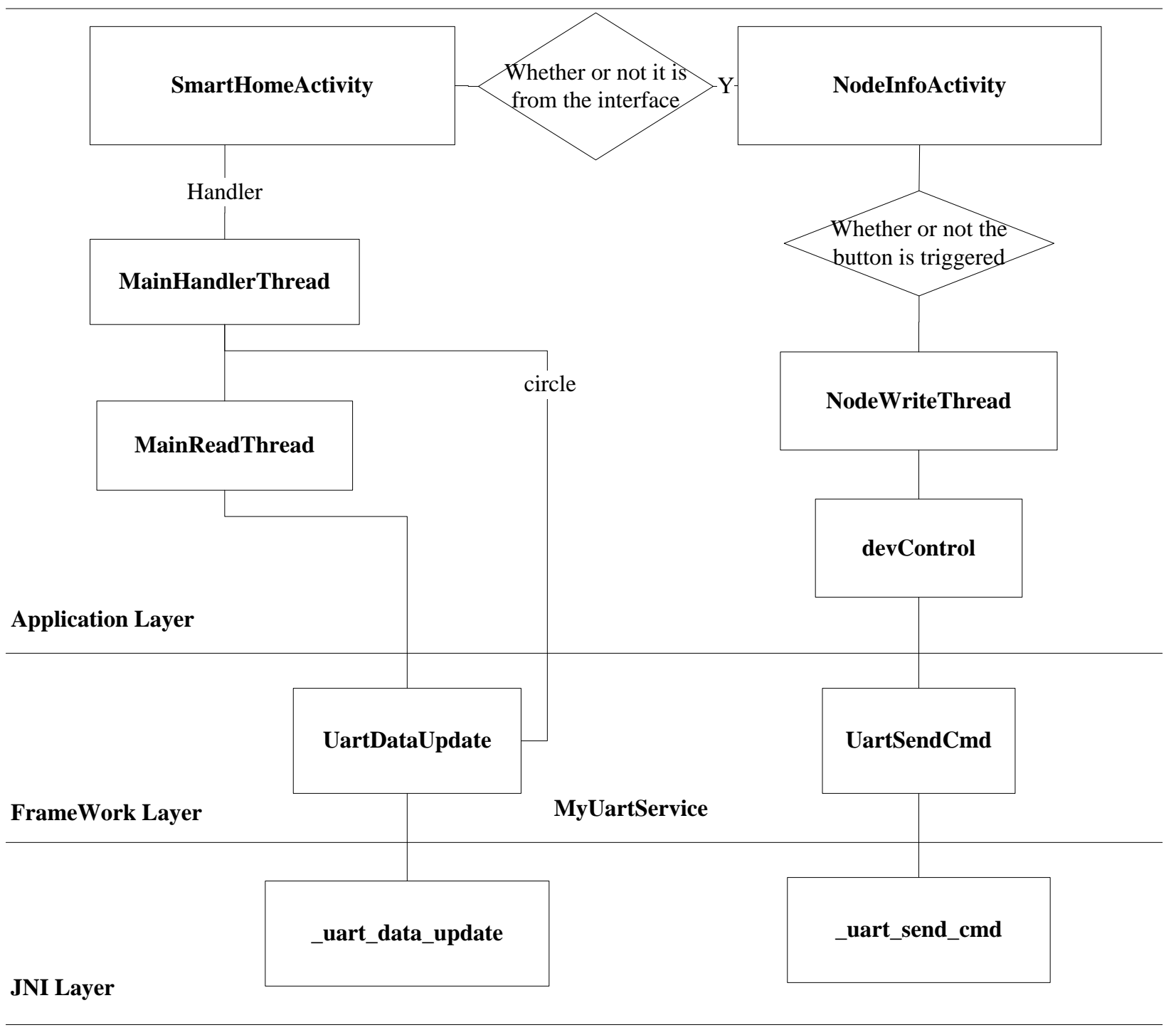

Figure 1. The flowchart of program design

\section{Designing Process}

A microcontroller is an integration of a central processing unit, memory, a timer, and a variety of input/output interfaces on an integrated circuit chip. Arduino is an open source electronic prototype platform that is convenient, flexible and easy to use. It is currently one of the most widely-used microcontroller. Comparing to the traditional 51 microcontroller, Arduino is more advanced and easier 
to master and more intuitive. Therefore, it is more feasible to design an environmental monitoring system based on Arduino platform.

Temperature Monitoring. In the measurement of temperature and humidity[6,7], DHT22 (AM2302) digital temperature and humidity sensor was applied. The sensor served as a temperature-humidity composite sensor with calibrated digital signal output, and high precision and excellent long-term stability. The sensor included a capacitive moisture sensing element and an NTC temperature measuring element, and was connected to a high-performance 8-bit microcontroller. The sensor had two power pins and a single bus interface port was used to transfer the data acquired to the microcontroller. The data sent through the bus interface consists of 16-bit humidity data, 16-bit temperature data (degrees Celsius) and 8-bit parity. They were then converted into the decimals and stored as float variables. After data acquisition is completed, the data was sent to the host machine via Bluetooth communication by Arduino. The DHT22 required a power supply of $3.3 \mathrm{~V}$ to $6 \mathrm{~V}$. After the sensor is powered up, its function is suspended for $1 \mathrm{~s}$ to initialize, hence any instruction or command should to not be sent to it during this period in order to prevent any data acquisition or communication failures.

Wind Speed Monitoring. In terms of wind speed measurement[8,9], an industrial wind speed sensor, RS485, was introduced. It can continuously monitor wind speed of its current environment and transfer its data to the host machine via bus interface. At the same time, converting the signal into MODBUS-RTU standard RS485 electrical signal and transmits it to the MCU. By coding the Arduino platfor, the output of the original $0-5 \mathrm{~V}$ signal from the anemometer is converted into wind speed ranging from $0-30 \mathrm{~m} / \mathrm{s}$.

External Display. HT16K33 mixly 8x8 LED dot matrix was introduced as the external image display module. The dot matrix module uses the HT16K33 chip as the driver, capable of running the module using only two I/O pins. By applying Adafruit_GFX and Adafruit_LED two library files in Arduino IDE and converting the collected data from float to char, the corresponding function of rolling text display output can be achieved on the LED dot matrix.

Host Machine Communication. In terms of communication, 1sheeld extension module for Arduino MCU was applied. This board has the function of Bluetooth communication and can transfer the data collected by the MCU to the host machine with Android operating system at a bit rate of $9600 \mathrm{kbps}$, by calling the development library file corresponding to 1 sheeld. This article implements an outdoor environment coefficient collection system that integrates data collection, system encryption, system presetting, and data collation.

Voice Recognition. In terms of voice recognition[10], the voice command is given to and translated, and transferred as 16-bit data via the host machine, for MCU platforms like Arduino lacks the processing capability to handle voice command. In this case, the advantages of the 1 sheeld extension module was fully demonstrated, for it was can complete offers the voice recognition files in its sources code library and was capable of fulfilling both the task of sending the collected data to the host machine and receiving the command data given by it, hence with proper coding, the function of voice recognition was achieved.

Home Appliances. Arduino platform had multiple PWM output interface, hence it was capable of giving out PWM signals to drive small electrical appliances. However, in order to control high power home appliances such as fans and lights, signal amplifier running on external power sources should be applied. As voice command given via the host machine was received by Arduino, the specific PWM signal would also be given out via Arduino I/O interface to drive the target home appliances.

\section{Summary}

In view of the problem that the environmental instrument detection parameters are too single, the reaction time is long, and the data is inaccurate, this paper proposes to use the Arduino MCU and its input and output expansion modules to program and combine the external circuit design to monitor the current environmental multiple parameters. Through Bluetooth communication, the information is converted into specific values and fed back to the Android system's mobile phone as a host computer. At 
the same time, the PC is integrated into the monitoring process, so that the single-chip microcomputer can feed back a plurality of parameter monitoring data to the computer in real time to realize feedback on the overall environment. The multi-parameter environmental monitoring instrument can simultaneously monitor multiple parameters such as temperature, temperature, and wind speed, and can return the data of the monitoring object in real time through serial communication. Simultaneously, it has features such as simple operation and portability, and can be widely applied in many fields. Environmental parameters monitoring and protection.

\section{Acknowledgements}

This work was supported in part by Liaoning Province Education Science " The 13th Five-Year Plan" project under Grant No. JG17DB354, JG17DB343, JG17DB355 and JG16DB523 and the educational reform project of the Liaoning provincial education department in 2016 under Grant 637 and 638.

\section{References}

[1] Jacobsson A, Boldt M, Carlsson B. A risk analysis of a smart home automation system[J]. Future Generation Computer Systems, 2016, 56(C):719-733.

[2] Lee Y T, Hsiao W H, Huang C M, et al. An integrated cloud-based smart home management system with community hierarchy[J]. IEEE Transactions on Consumer Electronics, 2016, 62(1):1-9.

[3] Khan M, Silva B N, Han K. Internet of Things Based Energy Aware Smart Home Control System[J]. IEEE Access, 2016, 4(99):7556-7566.

[4] Chen J, Yunyi X U, Wang Y, et al. Discussion on relevant technical indexes of automatic integrated multi-parameters water quality on-line monitoring system[J]. Water Resources \& Hydropower Engineering, 2011, 42(2):83-85.

[5] Yang W, Zhang D. Multi-parameters monitoring underground coal mine environment using mesh-structured wireless sensor networks[J]. Journal of Huazhong University of Science \& Technology, 2010, 38(10):70-74.

[6] Ma G M, Wu Z, Zhou H Y, et al. A Wireless and Passive Online Temperature Monitoring System for GIS Based on Surface-Acoustic-Wave Sensor[J]. IEEE Transactions on Power Delivery, 2016, 31(3):1270-1280.

[7] Amendola S, Bovesecchi G, Palombi A, et al. Design, Calibration and Experimentation of an Epidermal RFID Sensor for Remote Temperature Monitoring[J]. IEEE Sensors Journal, 2016, 16(19):7250-7257.

[8] Wakui T, Yokoyama R. Wind speed sensorless performance monitoring based on operating behavior for stand-alone vertical axis wind turbine[J]. Renewable Energy, 2013, 53(9):49-59.

[9] Comanducci G, Ubertini F, Materazzi A L. Structural health monitoring of suspension bridges with features affected by changing wind speed[J]. Journal of Wind Engineering \& Industrial Aerodynamics, 2015, 141:12-26.

[10] Caute A, Woolf C. Using voice recognition software to improve communicative writing and social participation in an individual with severe acquired dysgraphia: an experimental single-case therapy study[J]. Aphasiology, 2016, 30(2-3):245-268. 